\title{
De la guerre nomade : sept approches du conflit
} autour de la Côte d'Ivoire

\section{Michel Galy}

\section{(2) OpenEdition}

1 Journals

\section{Édition électronique}

URL : http://journals.openedition.org/conflits/1598

DOI : $10.4000 /$ conflits. 1598

ISSN : $1777-5345$

Éditeur :

CCLS - Centre d'études sur les conflits lilberté et sécurité, L'Harmattan

\section{Édition imprimée}

Date de publication : 1 septembre 2004

Pagination : 163-196

ISBN : 2-7475-7583-7

ISSN : 1157-996X

\section{Référence électronique}

Michel Galy, «De la guerre nomade : sept approches du conflit autour de la Côte d'Ivoire », Cultures \& Conflits [En ligne], 55 | automne 2004, mis en ligne le 08 janvier 2010, consulté le 30 mars 2021. URL http://journals.openedition.org/conflits/1598; DOI : https://doi.org/10.4000/conflits. 1598

Ce document a été généré automatiquement le 30 mars 2021.

Creative Commons License 


\title{
De la guerre nomade : sept approches du conflit autour de la Côte d'Ivoire
}

\author{
Michel Galy
}

1 Déconstruction des Etats, nomadisme guerrier, apparition de nouvelles formations politiques où se reforge l'ethnicité : depuis une quinzaine d'années, surgissent dans le Golfe de Guinée des phénomènes politiques qu'il convient de penser par delà - ou malgré - les violences exercées. Des analyses hâtives laissent entrevoir plusieurs écueils : s'enfermer dans un africanisme désuet qui se bornerait à l'évènementiel d'un seul pays (et la frontière linguistique, franco-anglaise semble, pour la recherche, plus marquée que celle des territoires...) et ignorerait la polémologie ; s'en tenir à des thèses fixistes (voire révisionnistes...) sur les ethnies et la violence endémique, ou sur « l'effondrement » d'Etats bientôt supposés délinquants - voire terroristes - que l'on verrait bien reconstruire et remodeler, selon un certain air du temps.

2 En revanche, une politologie informée de l'histoire des sociétés segmentaires, de leur anthropologie et de la sociologie d'Etats- Nations en interaction constante pourrait fonder une approche constructiviste de ces conflits - dont une approche comparative avec la région des Grands Lacs et la Corne de l'Afrique permettrait ultérieurement de dégager des constantes. Occasion aussi de reprendre les thèses clastriennes, mises à l'essai dans des sociétés paysannes, et de réfléchir sur les communautés et la violence, le lien et la décomposition du socius, mais aussi peut-être la paix à venir.

3 Impossibilité de la théorie à rendre compte de ces guerres nouvelles, comme en retard sur les phénomènes conflictuels qui naissent ou renaissent après la Guerre Froide ? Les apories de la recherche, parfois acharnées, ne résolvent rien: phénomènes plutôt nouveaux ou plutôt en continuité avec les sociétés anciennes? Dynamiques de relations internationales ou du « dedans » des Etats, du haut ou du bas? Conflits de la «politique du ventre » pour les rentiers de l'Etat et de « l'avidité des rebelles »? Et si, à l'inverse, par le biais de ce "Grand Détour », de ce terrible laboratoire politologique des conflits d'Afrique, se dégageaient des schèmes nouveaux sur la fondation des Etats et sur la 
place méconnue des communautés ou bien des recompositions sociétales inédites durant la guerre mettant à mal les idéo-logiques ordinaires (femmes combattantes, enfants-guerriers, etc.) ? Comment vivre "sans Etat »? Quel est le jeu de l'ethnicité et des identités durant ces conflits, la place du religieux et les formes de la violence?

4 A ces questions fondamentales que posent des "systèmes de guerre " comme celui de l'arc de crise ouest-africain, peu de réponses, que ce soit du côté de la phénoménologie comparée ou de la théorie interprétatrice. Une rare, ambitieuse et paradoxale tentative est celle de Paul Richards, spécialiste de la Sierra Leone en guerre. Dans des textes récents, Richards trouve dans les marginalités - ou plutôt dans les marginalisations sociales - la clef principale des conflits : celle à la fois de la jeunesse (focus de son ouvrage principal ${ }^{1}$ ) et des exclus de l'Etat ( natives »de Sierra Leone et du Libéria, par opposition aux élites «crio» et «congo »), auxquelles j'ajouterai personnellement les marges, les périphéries du territoire, notamment aux frontières, dans une perspective géopolitique (en opposition, bien sûr, aux Côtes et aux capitales surpeuplées et extraverties, tournées vers l'Océan). A cela, on ne peut qu'acquiescer, sans y voir d'ailleurs une quelconque surdétermination démographique vers la guerre qui frôlerait dangereusement la sociobiologie; mais faut-il prendre au sérieux la régression théorique vers l'anomie durkheimienne pour expliquer la guerre comme ce trou noir de la société, quand les règles ordinaires s'effondrent - alors que Richards établit, par ailleurs, les logiques des sociétés en guerre et les raisons des mouvements rebelles comme le $\mathrm{RUF}^{2}$, contre ceux qui voudraient uniquement y voir des organisations criminelles?

5 Peut-être faut-il voir dans ce paradoxal hommage au fondateur français de la sociologie un des traits d'humour typiquement britannique dont on soupçonne Richards d'user comme mécanisme défensif. Quitte à tomber sous une critique inverse, peut-être faudrait-il chercher du côté des thèses de Gregory Bateson pour rendre compte, non seulement de l'évolution des situations guerrières des individus par rapport à leur propre société, mais aussi de la dynamique globale, entropique et élargie, du système de conflit lui-même et du lien entre les deux. On sait que c'est justement à propos de la société Iatmul ${ }^{3}$ que Bateson proposa sa conception de la schismogénèse due à une «différenciation complémentaire » entre deux groupes, qui pourraient se retrouver ici au niveau des élites occidentalisées et des "natives", mais aussi du conflit latent «autochtone/allogène» qui les recoupe, agissant à micro-échelle. La combinatoire avec des différenciations symétriques, participant de la segmentarité ordinaire, comme l'opposition Gyo vs Krahn au Libéria, expliquerait le caractère entropique, et sans cesse renouvelé, des conflits en ligues et en damiers, qui passeraient les frontières pour devenir transnationaux.

6 Au-delà de ces idéo-logiques reconstituées sur un fond de violences croissantes, une déterritorialisation continue aboutirait à un nomadisme croissant et à une atomisation des groupes guerriers ayant trouvé, dans la mouvance et la prédation, un mode de vie nouveau. La guerre, elle-même, semble alors nomadiser, en fonction des sorties de crise et des paix provisoires, pour se porter vers de nouveaux pays, territoires à prendre pour les factions des anciennes guérillas antagonistes - comme la Guinée ou la Côte d'Ivoire l'ont été temporairement pour des combattants issus du Liberia ou de la Sierra Leone. Suivant Clastres dans sa description de l'ambiguïé du pouvoir guerrier qui défend et menace à la fois les communautés comme pôle de pouvoir accumulatif, incontrôlé et destructeur, Gilles Deleuze montre bien l'opposition fondamentale entre 
l'Etat et cette "machine nomade» qui déterritorialise et recompose les sociétés, réinventant les formes d'hybridation des techniques de guerre, de la violence et du symbolique 4 . C'est le cas des réutilisations des $4 \mathrm{X} 4$ dans la blitzkrieg tchadienne, de l'usage de la sorcellerie d'attaque ou de défense, du renouveau de l'esclavage ou du dépeçage libérien du corps de l'ennemi, sur le modèle de la chasse et du gibier...

On concevra ainsi, autour du conflit ivoirien, en deçà de la violence guerrière et au-delà des frontières, des résonances ouest-africaines ou, au contraire, les jeux d'une microethnicité sans cesse recomposée, comme une série de plateaux ou d'intensités qui lui font écho et l'informent en retour. En deçà de l'Etat, en effet, se construisent des machines et des tensions à base anthropologique, puis tout un «secteur informel du politique » culminant, en temps de guerre, dans une pathologie du pouvoir et de la violence qui n'est pas sans racines, contrairement à ce qu'affirment les rares échos médiatiques. Réinterrogeant les catégories traditionnelles du politique, l'analyse de la guerre nomade ouest-africaine est si peu orpheline que ses conclusions vaudraient pour la région des Lacs et la Corne de l'Afrique, pour des catégories sociétales - et donc guerrières - sensiblement différentes.

D'une phénoménologie à une topologie des développements de la guerre nomade prise comme un système, un champ conflictuel, on induira ses mécanismes d'expansion ; mais seule une généalogie comparée des formes du pouvoir - au-delà, en deçà des Etats - permettra de donner sens aux formes du conflit, en particulier ivoirien. Face à la multiplication des pseudo analyses se bornant à un descriptif de l'évènementiel, on prendra sans risque le choix inverse : réfléchir au système de gouvernance mondial (aux causes de ses échecs, aux obscures rébellions infraétatiques) en interrogeant autant les communautés que l'ethnicité; aux figures locales de la violence et du pouvoir (Maître de terre, Maître des gens, Maître de guerre); aux procès de déterritorialisation de la violence qui correspond in fine aux errances des guerriers et aux conquêtes de leurs dieux; enfin, aux médiations permanentes, espoir d'une régulation nouvelle.

\section{Machines néo- segmentaires}

9 Ce n'est plus le Liberia, c'est la Sierra Leone. C'est la Côte d'Ivoire, ce n'est plus elle. Demain, la Guinée ? Et même, ce n'est plus Abidjan, Bouaké, mais Ouagadougou, Dakar, Accra... Peut-être Paris, Londres, Washington. Plus que des Etats, des sociétés, des services, des clients, en liens complexes avec des lieux de pouvoir. Cette guerre nomade, décidément, déterritorialise de toutes parts.

Guérillas africaines, conflits de basse intensité? Plus que l'épopée surréaliste, catastrophique d'un Guevara aux prises avec l'africanité imprévue des insurrections congolaises et avec un Kabila jouisseur et retors ${ }^{5}$, c'est la trajectoire du plus américain des présidents africains, Charles Taylor, dont il faudrait peut-être scruter l'itinéraire pour comprendre ces nouveaux conflits. Évènement fondateur ou origine que ce jour de décembre 1989 où, à la tête d'une centaine de rebelles armés par le Burkina et financés par la Libye, ce "Maître de guerre " franchit la frontière de la Côte d'Ivoire vers le Libéria. Son parcours corrobore l'image du politique «traducteur» de registres et d'univers : mi-congo, mi-gyo, homme d'affaires douteuses, guerrier, administrateur et politique, usant des discours de la magie et du religieux comme de simulacres électifs et représentatifs. 
11 Les lieux marginaux où s'origine ce long et sanglant conflit ne sont pas sans rappeler ces périphéries où Vidal Naquet voyait le «Chasseur Noir" mener ses errances obscures jusqu'à déstabiliser la Cité 6 . Ces contreforts du Mont Nimba, ces zones forestières et montagneuses des guerriers Yacouba, à la réputation ambiguë, sont à réfléchir comme le théâtre initial d'un conflit entropique - exemplaire, peut-être, dans le sens d'un affrontement de la violence extrême d'une armée d'Etat avec la violence exacerbée d'une société segmentaire.

12 Là apparaît aussi ce phénomène de résonance, ce point de fusion de deux types de violence autochtones : celle, segmentaire, de longue durée, branchée sur les complexes alliances ou inimitiés de l'ethnicité ; celle, plus nomade dans ses parcours et en même temps toute entière tournée vers la conquête du centre, et du pouvoir d'Etat, des nouvelles formes de guérilla. Et c'est d'ailleurs une des caractéristiques majeures des guerres nomades que cette circulation élargie, permanente, inextricable, des enjeux et des violences entre les trois niveaux de l'ethnicité, du national et des relations internationales.

13 Quand les sbires de l'armée du président Samuel Doe massacrèrent, en 1990, les villageois Gyo et Mano du Comté de Nimba sans distinction d'âge et de sexe en déclarant cyniquement que cette manière de faire venait de "l'impossibilité de distinguer les rebelles de la population", ils fondaient aussi le principe de ces "guerres néosegmentaires ", qui impliquent peuples et Etats sur le modèle autochtone, mais élargi, d'un conflit total hors rituel et, même, hors symbolisme, bien éloigné des guerres limitées. Pour reprendre Clastres, dans ce type de "sociétés paysannes" les communautés ont l'expérience réelle de la violence d'Etat, qu'elles ne font que pressentir dans les « sociétés sauvages », et leur refus, conjuration et lutte n'en est que plus inexpiable.

14 Cette machine néo-segmentaire joue sur un modèle structural sous-jacent, qui s'actualise dans le conflit et produit de nouveaux agencements d'ethnicité : il en est ainsi du conflit du $19^{\text {ème }}$ siècle entre Krahn (ethnie de Samuel Doe) et Gyo (dont est issue la mère de Charles Taylor); de l'alliance des premiers avec les Madigos, qui ont servi de milice exterminatrice dans le Comté de Nimba; Madigos eux-mêmes opposés aux autochtones du Comté de Lofa, dans le conflit en cours. Toutes ces alliances et oppositions en mosaïques, déclinées sur le territoire et par delà les frontières, pourraient être rapprochées des «ligues » (voir les «leffs » berbères) d'Afrique du Nord.

15 Un des effets de ces conflits serait de multiplier les niveaux spatiaux et structuraux : en particulier le langage de la guerre-ouest africaine se décline autour de l'articulation secondaire opposant «natives » vs « congo » au Liberia (ou «crios » de Freetown pour la Sierra Leone). On peut repérer des passages, ou des seuils, à partir d'évènements particuliers, qui font en quelque sorte passer le conflit à un niveau de violence plus important et à une reproduction élargie : c'est ainsi que l'implication des Madigos islamisés, comme supplétifs de Doe, conduisit le NPLF ${ }^{7}$ de Taylor et ses alliés à des massacres anti-musulmans à l'échelle du pays - et, de la part de ces derniers, à une solidarité en retour sur cette base ethnico-religieuse. A l'inverse, l'opposition des Madigos aux Lormas du Comté de Nimba se retraduit par ce pattern plus profond du politique : autochtone vs allogènes, premiers arrivants vs derniers arrivants. 


\section{Liberia, Etat-de-guerre}

16 Au nomadisme guerrier correspondraient des changements, glissements, mouvances de l'Etat qui, comme en écho, se transformerait insidieusement en autre chose, subverti par un appareil de guerre qui le précède, alors tout autant machination voulue que machine perverse. Les «qualités » d'un Maitre de guerre sont, comme pour un leader politique, de conjuguer au mieux les registres et les institutions : ici les appareils de guerre et les discours de l'ethnicité.

17 Fasciné par les USA - au point de connaître leurs geôles - et pourtant renié («le Milosevitch de l'Afrique " pour le Département d'Etat), Charles Taylor a joué de ses multiples appartenances mais a aussi travesti sa Conquête en victoire électorale, tandis qu'il investissait et transformait des structures d'Etat déliquescentes, dans un processus qui mérite d'être analysé de plus près. La politologie, africaniste ou non, a esquivé en effet l'étude des avatars politiques sous la guerre, qui se constitueraient volontiers pourtant, dans une conception d'anthropologie dynamique, en outil essentiel pour comprendre les origines, structures et changements de l'Etat.

Mais peut-être faudrait-il partir, auparavant, de ce que l'on pourrait nommer les «mouvances ordinaires de l'Etat»; ce changement interne, permanent de l'Etat ne se traduit que marginalement par des changements de personnes, pertinents seulement en tant qu'ils signifient des modifications de forces et de positions, voire d'institutions. De fait, des pans entiers des appareils sont parfois plus ou moins subtilement modifiés, tandis que l'apparence semble quasi-identique. J'ai analysé ailleurs les "avatars de la DCGTX ", agence ivoirienne de grands travaux dépendant de la présidence, devenant un "gouvernement-bis » de l'ombre, par lequel le pouvoir blanc vidait de son sens les appareils ministériels - et le système représentatif, par ailleurs déjà truqué. La suppression des fameuses "sociétés d'état » (SODE) en fut symétrique, en ce sens que les barons qui les dirigeaient y avaient trouvé les moyens de leur politique néopatrimoniale et s'y étaient taillé des « fiefs » qui menaçaient Houphouët Boigny ${ }^{8}$.

19 Par rapport à ce qu'on a pu décrire comme un "caciquisme urbanisé ", quel est le diagramme des pouvoirs dans un Etat-de-guerre? Au Libéria, tout se passe comme si ressurgissait derrière les appareils officiels et son système électif, quelque chose comme un simulacre d'Etat, en un sens le fantôme de l'organisation du NPLF, appareil de Conquête du Taylorland. Territoire alors restreint à la capitale par diverses rebellions (notamment le LURD', soutenu par la Guinée et les USA) où l'Etat, quasi virtuel, atteint le paroxysme de ses transformations, plus un simulacre qu'un « quasiEtat » : un « Etat-pour-la-guerre », extension machinique du guerrier clastrien.

D'un côté, l'Etat-de-guerre se décline sur un mode orwélien, où, dans la novlangue internationale en cours, la propagande et le signifiant lui-même désignent l'inverse du réel : l'institution des "forces de sécurité » omniprésente, qui « doublonne » l'armée, est désignée par le vocable d' « anti-terrorist unit» (ATU) et constitue la garde de fer de Taylor. Cette unité prétorienne, toute de noir vêtue, semble à la population, par ses privilèges et ses exactions, plus proche de sections spéciales que de commandos légalistes... Rôle ailleurs tenu par les gardes présidentielles ou les milices ethniques qui représentent le cœur du pouvoir - y compris au besoin contre l'armée, ou ce qu'il en reste. 
rapports sociaux fondés sur l'esclavage, et les formes de violence les plus extrêmes : exécutions sommaires, dépeçages, recours multiplié à la sorcellerie, expérimentation macabre et sexuelle... Homologie entre la fin des rituels et la recherche perverse de la limite, dans une terrifiante ingéniosité qui recourt volontiers aux fantasmes, mythes et légendes du vieux fond culturel, pour les faire émerger dans l'insoutenable horreur du Réel.

\section{Du Non-Etat au Sur-Etat : Sierra Leone, un pays «border line »}

Suivant ici Pierre Legendre ${ }^{10}$, on pourrait avancer que la politologie sierra léonaise relève surtout d'une pathologie du pouvoir : de l'Etat - ou de ce qui en tient lieu; des communautés, et de leurs conflits internes; des violences et des institutions - et de leur refoulé. Analysable en termes d'évènements, mais encore plus en forme d'instances psychopolitiques, le pays présente à l'observation des "formations pathologiques » symétriques et spatialisées, alternantes et polarisées. Après avoir observé, sur le terrain, deux ethnies frontalières, et constaté l'ampleur des traumatismes de l'après conflit (ou peut-être de la trêve avant le prochain conflit), mais aussi de la violence sousjacente, nous avons proposé de conceptualiser ces sociétés de l' «entre-deux» comme 
fonctionnant sur le mode du «comme si » (as if), situation éminemment précaire et révocable, sur le fil du rasoir.

Il serait temps, en Sierra Leone et aussi en Somalie ou au Congo, en bien des lieux, mais surtout aux frontières, de commencer à analyser le Non-Etat ${ }^{11}$. Pas seulement en termes de négativité, ni - plus subtilement - comme retour des formes politiques anciennes (communauté, segmentarité...), mais comme possible, hic et nunc, d'autres formes du politique, seulement aujourd'hui esquissées. Il serait temps de comprendre tant les agencements des pouvoirs internes, notamment communautaires (et clivés selon l'opposition structurale Aînés/cadets), que l'articulation avec les instances ou les factions étatiques, perçues justement comme incarnation d'une Aînesse sociale qui aurait rompu le pacte implicite du socius : redistribution accompagnant la prédation, alternance spatiale et mobilité sociale contre acceptation de la violence fondatrice.

Le Non-Etat des zones frontalières, là où le pouvoir de la capitale s'affaiblit ou disparaît, n'est pas une zone anarchique, mais une recomposition des structures sociales préexistantes, dans un système de guerre élargi. On ne reviendra pas ici sur l'ampleur des trafics - d'ailleurs très mobiles - où des auteurs comme Gberie ${ }^{12}$ voient, à tort, les racines du conflit alors qu'ils ne sont que les moyens du pouvoir, y compris d'Etat, comme les diamants de la région de Koïdu.

En revanche, on peut distinguer trois phénomènes régulateurs de ces espacesfrontières, autour de l'instrumentalisation (plus que de la négation) de cette ligne - en tout sens - relevant de l'imaginaire. La force des échanges, fluctuants et dynamiques, qui jouent en quelque sorte du «différentiel étatique » - qui n'est pas que monétaire; le contrôle des hommes et des zones d'extraction, effectivement efficace pour se procurer des armes et/ou des devises et étendre son territoire ; l'utilisation du système de l'Aide, et notamment des camps de réfugiés, selon des logiques autochtones.

On sait bien, dans des pays frontaliers d'une puissance régionale comme le Nigeria, que les mille petits métiers liés à la frontière sont capables de s'inverser du jour au lendemain, les pays périphériques devenant des sortes d' "Etats- entrepôts", selon l'expression de John Igué $^{13}$ dans le cas du Bénin. Même chose ici, à une échelle plus réduite, et cette activité porte principalement sur les matières premières principales, agricoles ou autres (diamants, caoutchouc...), des pays en guerre, transférées aux pays limitrophes - trafic d'ampleur, souvent aux mains des diaspora, des grands commerçants africains en réseaux ethniques (de type mouride), mafia, rébellions ou armées « nationales ». Au-delà d'une perspective très onusienne, et souvent artificielle, de reconstruction et de société post-conflit, les sociétés locales se réorganisent spontanément par d'actives micro-transactions, notamment autour de réseaux féminins qui tissent de nombreux liens transfrontaliers, comme lors des marchés kissi, par exemple, au nord-est de la Sierra Leone; et bien plus loin: rappelons que cette ethnie se trouve aussi en Guinée et au Libéria.

A l'inverse de la problématique étatiste, dont nous avons montré à quel point elle est inadaptée et décalée, aucune réflexion sérieuse n'est faite par les pays occidentaux ni par les organisations internationales (et pas davantage par les ONG) en termes de gouvernementalité, de pouvoirs autres que l'Etat, justement. C'est plutôt une caricature d'Etat néocolonial, un retour au $19^{\text {ème }}$ siècle aggravé de tous les défauts onusiens qui est imposé au pays: une sorte de dangereux «Sur-Etat» (comme un terrible Surmoi institutionnalisé), qui refoulerait - jusqu'à quand ? - toutes les révoltes et les formes de pouvoir autochtones. 
30 Et pour les «natives » sierra léonais (le point de vue des crios de Freetown est peut-être différent, et encore...), c'est bien un même pouvoir répressif multiforme qui réprime et discipline - en tout sens: ONU, forces britanniques infiltrant et dirigeant le gouvernement de l'Etat fantoche, projets multi- et bilatéraux, ONG, etc. Leur habitus somptuaire en font bien les héritiers de ce "métier de Seigneur» des Officiers des Affaires Indigènes: villas, avions, $4 \mathrm{x} 4$ et climatisation, ordinateurs dernier cri et Internet en sont simplement les nouveaux emblèmes, ceux des représentants de l'Empire mettant au pas la Multitude, sur fond de grand dénuement mais sous le signe de la technologie triomphante.

\section{Communautés vs Etats?}

31 Pourtant, il existe bien des formes de gouvernementalité, communautaire entre autres, négligées par les politologues, et curieusement déniées par d'éminents africanistes. Même ancien, même colonial, l'Etat en Afrique s'est toujours heurté à d'autres légitimités qu'on aurait tort de résumer à la seule ethnicité, dont on précisera plus loin dans quels dispositifs ce niveau d'identification entre en rapport dans les régimes du politique. C'est dire à quel point la pseudo-problématique des Etats "faillis", " fantômes ", " effondrés » - et autres métaphores dont la multiplication même montre bien l'inadaptation - vient d'un référent occidental de gouvernance échappant d'ailleurs à toute critique historienne.

Une relecture des thèses de Clastres pour les "sociétés paysannes" comme celles d'Afrique de l'Ouest amène à analyser une certaine variété des formes du politique à l'échelle locale, dans lesquelles l'instance communautaire prend un aspect et une pertinence sensiblement différents. Si l'on retient l'opposition idéaltypique proposée par Clastres (qui n'est nullement exclusive de différenciation communautaire interne, ni d'autres instances intermédiaires du pouvoir), la communauté fait plus que pressentir l'Etat: elle a affaire à lui, "malencontre » majeure, nous dit la Boétie, et d'abord par la rencontre même de la guerre étatique pour le contrôle de ses propres communautés.

33 Mais de manière différentielle : selon que l'on se retrouve, pour résumer le propos et simplifier des évolutions historiques complexes (émergence d'Empires et de Royaumes éphémères, depuis le Mandingue à celui de Samory, ou de Kong, avec autant de constructions et de déconstructions de leurs appareils d'Etat...), en zone segmentaire de pouvoir diffus ou à l'inverse en pays de pouvoir fort et d'Etats traditionnels, dans des lieux de communautés autonomes ou à l'inverse soumises. Toute analyse du politique contemporain devrait donc passer par un minutieux inventaire du politique précolonial, une géopolitique de leurs pouvoirs pluriels.

34 En effet, pour la Côte d'Ivoire, dès l'époque pré-coloniale, les Etats "traditionnels » occupent des espaces spécifiques, entre lesquels subsistent tant bien que mal d'autres formes d'organisation sociale. La "forme étatique » - comme les ethnies - a aussi une histoire, et une géographie. En effet, on assiste à une véritable migration de l'Etat depuis le Ghana et ses prestigieux royaumes ashantis, de l'Est vers l'Ouest, jusqu'au fleuve Bandama : c'est ce que traduisent beaucoup de récits de migration, tel celui de la reine Abla Pokou chez les Baoulé. 

villageoise est souvent l'unité de base, sur laquelle s'établissent les grandes chefferies, les Royaumes, les Empires. Bien qu'elle soit parfois, elle-même, socialement et ethniquement composite, elle constitue souvent le lieu de prélèvement collectif du tribut ou de l'impôt, qui en un sens la renforce comme collectivité. En d'autres termes, jusqu'à aujourd'hui, Etat et communauté, malgré leur opposition fondamentale, trouvent des modus vivendi qui les confortent : à l'étatisation progressive correspond une communautarisation qui passe d'abord par la fixation au sol. A l'opposé, de nombreux peuples s'organisent intentionnellement «contre l'Etat», refusant tout contrôle et tout pouvoir coercitif, qu'il soit parmi eux ou extérieur, traditionnel et contemporain - ce qui explique les formes de la violence et de la guerre dans l'Ouest segmentaire, en continuité culturelle avec le Libéria.

tialement, on peut considérer les zones de pouvoir segmentaire : peuples Krou, Lobi... comme l'hinterland des royaumes, lieux de rezzous, de guerres, de conquêtes et de pillages pour les Etats traditionnels qui les entouraient. A l'époque contemporaine, la manipulation de mouvements d'opposants (MPCI pour le pouvoir de Ouagadougou, MODEL ${ }^{14}$ pour celui d'Abidjan) participe de cette logique : mener le conflit plus loin, dans ces zones de pouvoir diffus ou effondré.

37 A l'inverse, la zone Nordest très intéressante à considérer, sur le plan théorique d'une réorganisation des pouvoirs coutumiers - et non sans contradictions: pays de chefferies et marqué par le système Poro, le fort pouvoir communautaire s'allie à la reconnaissance de "grandes familles", urbanisées, qui exercent les fonctions de médiation et de choix politiques, en interface avec la société nationale. En continuité avec les sociétés du Mali et du Burkina, elles sont marquées par un progrès de l'islamisation, contre les cultes sénoufo en particulier. Ayant gardé un mauvais souvenir de la conquête de Samory, elles passaient pour soumises aux pouvoirs extérieurs, colonial puis national.

Le micro-politique communautaire, lui aussi, a une Histoire, pas si mineure qu'il y paraît : participant de cette pluralité des pouvoirs et même des légitimités, il signifie bien souvent une opposition radicale de principe (même s'il compose au quotidien), avec les appareils d'Etat, assurant depuis longtemps ce que l'anthropologue Ernest Gellner ${ }^{15}$ nommait justement, pour une autre aire culturelle, « une auto-administration clandestine ». C'est en quoi les thèses néo-fonctionnalistes de "l'empilement des pouvoirs locaux » sont limitées dans le temps et l'espace. Le pouvoir n'est pas unique dans les communautés, mais pluriel : manière ordinaire de favoriser son contrôle.

C'est que le pouvoir communautaire reste fort, pôle structurellement opposé à celui de la chefferie. La fonction qui correspondait le plus à celui de l'actuel chef de village pourrait se traduire par «maitre des gens», dans un système de chefferies villageoises, tel le « kéféguefolo » sénoufo. Mais la fonction la plus importante, le pouvoir de l'ombre, est jusqu'à nos jours celui du chef, ou plus exactement du "maître de terre», en général le plus âgé des descendants en ligne directe de l'ancêtre fondateur: tel le «tarfolo» chez les Sénoufo. La guerre implique souvent une fonction en principe temporaire, celle du «maître de guerre » aux pouvoirs sacrés («mystiques », dit-on couramment aujourd'hui en Côte d'Ivoire) étendus.

La combinatoire des trois pouvoirs se rencontre: si des négociations évidentes se passent avec le chef de guerre durant le conflit, si le chef de village sert d'interface avec 
le pouvoir d'Etat, c'est avec le chef de terre que se négocient la paix, les rapports autochtones allogènes, les sacrifices et les pactes consécutifs.

En revanche, la confusion des trois pouvoirs ou leur emboîtement politico-spatial constituent un autre agencement: chez les Sénoufo, il arrive que le plus âgé des descendants de l'ancêtre-fondateur soit «le chef de village, le chef de terre, et le chef du bois sacré». Dans un contexte très différent, chez les Agni, Amon d'Aby montre que le patriarche (afilié- kpangni) chef du clan des descendants d'un ancêtre commun «est tout à la fois prêtre, juge, conservateur du patrimoine (sic) du clan, et chef politique ».Comme le pouvoir politique est emboîté, à plusieurs niveaux éventuels de l'espace politique ( « rois », chefs de canton ou de clan, chef de village ou de lignage...), la Maîtrise de terre peut concerner plusieurs espaces.

Selon les ethnies, de véritables prêtres de religions africaines peuvent coexister avec le Maître de terre; d'autres cas plus étonnants, comme le Bagnon bété ou krou, peuvent représenter l'excellence d'une culture au point de galvaniser les communautés. Mais l'opposition structurale à ces titulatures multiples du pouvoir communautaire se trouve du côté des idéo-logiques d'âge et de sexe : les jeunes et les femmes (comme les étrangers), quoique subordonnés, ont un pouvoir parallèle, particulièrement important, pour le côté féminin, dans les sociétés matrilinéaires de l'Est.

43 En général, l'idéal-type du pouvoir communautaire se retrouve dans une Assembléedélibérative de tous les chefs de familles, femmes, jeunes et étrangers assistant en groupes, et délibérant à leur tour de parole ; c'est de cette assemblée qu'est issue un Conseil, le plus souvent gérontocratique, qui assiste la ou les chefferies. C'est cette procédure qui induit l'illusion fréquente pour les observateurs extérieurs que «le pouvoir est aux vieux ». Les réalités sont infiniment plus complexes et que cela soit dans les conflits entre les communautés et les pouvoirs extérieurs, ou encore dans les différentes instances de la chefferie, ou bien au niveau des catégories internes, suivant les idéo-logiques sociales (femmes, jeunes - guerriers notamment -, étrangers). Cependant, l'opposition structurale et ternaire Assemblée/Conseil/Chefferie cède souvent à une opposition duelle, notamment celle marquée par la volonté tendancielle de la chefferie de s'autonomiser, et le désir contraire de la communauté de contrôler un «chef sans pouvoir ».

\section{Maîtrise de terre : le pouvoir de l'ombre}

44 En retrait du « Maître des gens »- qu'il soit chef de village ou non - se tient, dans les assemblées, le Maître de Terre, pouvoir souvent plus réel que le premier, équivalent structural des caciques méso-américains. Des auteurs comme Alain Marie ${ }^{16}$ s'essaient $^{2}$ en quelque sorte à transposer cette dualité et cette hiérarchie dans l'analyse du politique national, s'efforçant par là de contrer les néoculturalistes (comme les analyses en termes d'ethnonationalisme croissant et d'ethnicisation du conflit ivoirien), en voyant dans le foncier, et singulièrement la « faim de terres » dans le sud forestier, les clefs en dernière instance de la crise ivoirienne.

Pourtant il nous semble, après une longue recherche de terrain sur ces conflits dans les années 1990, que la plupart des analystes les plus spécialisés comme J.P. Chauveau pour 《la question foncière ${ }^{17}$ ou J.F. Bayart pour l'analyse fondamentale des rapports autochtones/ allogènes ${ }^{18}$ manquent la dimension proprement religieuse de l'alliance à la terre et de l'accueil des étrangers. Origine de toute chose, la Terre se dit une Déesse 
aussi bien qu'une réalité matérielle: chez les baoulé, Asye représente la divinité chtonienne comme asye (toujours munie d'attributs tels que la couleur, la forme...) correspond à la terre cultivable. Dans un texte d'une rare densité, Pierre Etienne ${ }^{19}$ analyse cette double Nature, dans une cosmogonie complexe où elle prend place entre les Dieux et les Ancêtres, peuplée de nombreux esprits et génies des lieux, acculturée par les « hommes forts » qui passent avec elle une alliance sacrée.

Dans une volonté louable de simplifier ce que nous avions nommé "l'imbroglio foncier ", des chercheurs comme J.P. Chauveau tendent à surinterpréter, ou plus exactement à surinstitutionnaliser, les relations entre maîtres de terre et allogènes, entre autochtone et exploitant, migrant sahélien ou ivoirien, selon des modalités sensiblement différentes, en y repérant l'institution d'un " tutorat » néo-coutumier ${ }^{20}$. Outre, en effet, que la maitrise sur une parcelle de terre peut être multiple et parfois contradictoire (voir Plan Foncier Rural, PFR infra), il s'agit plutôt d'un système interrelationnel (et interethnique) complexe et disputé, où jeu de rôles et négociation, violence symbolique ou réelle, recours multiples à des médiations, forment plus un champ de pouvoirs qu'une institution. Ainsi le primat de l'autochtonie explique bien des formes des conflits actuels : alors que les migrants sahéliens représentent trois à quatre millions de personnes dans le Sud forestier, et qu'ils sont souvent ultra- majoritaires dans certaines zones de l'Ouest (autour de la forêt de Taï, proche du Libéria, près de $95 \%$ des planteurs sont allogènes), les migrants, malgré des résistances ponctuelles, ont toujours choisi la fuite et l'exode.

Un phénomène complexe et méconnu de relations autour du foncier peut aussi expliquer en partie la situation actuelle: historiquement, l'Etat ivoirien a toujours défini sa politique locale contre le pouvoir de l'autochtonie - sauf à considérer le peuple baoulé comme le «Maître de terre » collectif du pays. On connaît l'adage célèbre du président Houphouët Boigny : «la terre appartient à celui qui la met en valeur ». On sait moins que les baoulé - dans les terroirs saturés dans lesquels se pose très peu le problème allogène, et où n'existe quasiment pas de marché de la terre l'interprétaient, dans les forêts de l'Ouest (en zone bété par exemple), comme une permission d'aller à la fois contre le droit national (forêts classées, biens domaniaux) et coutumier (maîtrise de terre).

Ce que traduisait clairement dans l'espace l'implantation baoulé, en "colonies de peuplement" autonomes (campements, puis villages), sorte de pôle tiers d'un "ethnoscape » ${ }^{21}$ organisé autour du village autochtone baoulé et du « double » urbain d'Abidjan. Toute autre est l'implantation nordiste, par exemple sénoufo ou mossi, qui constitue un «dyoulakro » (nom populaire des quartiers nordistes) juxtaposé au village autochtone, et donc implicitement dépendant. La prépondérance baoulé a donc coïncidé un temps avec l'idéologie étatique dans la volonté de "casser la chefferie », ce qui a pris un tour d'autant plus aigu que, comme dans bien des pays subsahariens, le colonisateur français (ou anglais, dans les pays limitrophes) a tenté d'instrumentaliser, à l'inverse, le prestige autochtone en un parti pro-colonial, frontalement opposé, au tournant des Indépendances, au PDCI-RDA ${ }^{22}$, dont on sait la victoire et l'hégémonie finale.

49 Au quotidien des appareils locaux d'Etat, l'ambiguïté restait des deux interprétations antagonistes des transactions foncières, par lesquelles un autochtone "prêtait » ou "vendait » des terres à un allogène. La première interprétation, conforme au code de l'autochtonie, veut qu'il s'agisse d'usufruit contre des prestations régulières. La seconde, 
symétrique, est celle des allogènes férus sans le savoir d'une pure conception de droit romain, qui disent avoir "acheté" une terre transmissible et s'appuient sur "le papier » : l'attestation délivrée à des titres très divers par des agents administratifs. Les agents de développement, occidentaux ou ivoiriens, ont pu conforter l'idéologie marchande (qui reste le but caché des projets fonciers comme le PFR, financé par l'AFD et la BM) en se servant d'un vocabulaire décalé, ignorant des réalités coutumières, tel que "propriétaire foncier » vs "exploitant », repris par le discours allogène dans une certaine "mauvaise foi » d'autant plus remarquable que les intéressés excluent ces conceptions européennes dès lors qu'il s'agit de leurs terroirs d'origine. Notons d'ailleurs que l'obligation de demande de terre auprès du maître de terre est toujours respectée en ce qui concerne les parcelles vivrières, annuelles: les problèmes récurrents, de "propriété » et d'héritage, se posent pour les terres de parcelles de rente (café, cacao, cocotiers, palmiers à huile, etc.).

Dans la conflictualité ordinaire autour du foncier que nous avons pu étudier sur le terrain, ces «néo-conflits» autochtones/allogènes s'inscrivent sur un fond de segmentarité foncièredont on a pu dessiner des figures récurrentes, servant de "patterns» aux conflits actuels, et expliquant bien des traits de la crise globale. En pleine zone baoulé, autour de Béoumi, la raréfaction des terres ravive les conflits frontaliers, interlignages ou intercommunautaires et même intertribaux. Des conflits internes nouveaux apparaissent, en particulier intergénérationnels: la jeunesse autochtone n'a parfois guère plus envie de respecter le " Maître de terre » autochtone que le travailleur migrant, mais entend parfois s'y substituer. Une des escroqueries classiques en milieu rural est d'ailleurs la «vente» par des jeunes villageois de terres autochtones à des migrants, les contractants étant le plus souvent complices...

51 Comme pour la prépondérance baoulé ou le "pacte de croissance implicite", la revendication allogène met à jour l'implicite des rapports sociaux - et donc politiques dans lesquels l'ivoirien en général, l'autochtone en particulier, se perçoit toujours comme privilégié d'une rente foncière collective, due à une dette perpétuelle de l'Etranger.

\section{Errances de Mars}

«Ils sont venus comme la destinée, sans égards, sans cause, sans prétexte...» : de même que la soumission et la servitude volontaire, pour la Boétie, a aussi bien des séductions celées, le beau texte cité par Deleuze ${ }^{23}$ relève la fascination du guerrier nomade, installé dans le mouvement, comme d'autres sont ancrés aux lieux, dans l'insoumission et la prédation, la consumation des biens et non leur accumulation. Dans cette guerre nomade, ce sont des groupes fluctuants de jeunes et de guerriers qui se font passeurs de frontières, créateurs de foyers insurrectionnels, conquérants de capitales. Et ce mouvement - à part leur mort toujours possible -, devient leur vie même, un mode de sociabilité, où bientôt, dans la guerre et l'errance, s'installent des sociétés toutes entières.

Mais pour autant, au-delà des anecdotes, évènements et volontés, on peut discerner des échelles, des vecteurs et des spatialités à ce mouvement nomade qui présente des temporalités propres.

Si on pouvait parler, sans essayisme péjoratif à la Kaplan ${ }^{24}$, de "guerres sauvages ", ce serait plutôt pour marquer la proximité avec les chasseurs, ces "palpeurs-de-brousse » 
et découvreurs, selon F. Héritier, venus des périphéries et des confins, pour s'en prendre à la Cité. Et tout à la fois sachant qu'ils représentent un modèle alternatif ethnique, communautaire, nomade, selon les cas et les époques, ou plutôt les agencements de ces trois états - au modèle classique, ancêtre de l'Etat. Comme si le drame des origines, de la déperdition et de la servitude (vs l'insurrection légitime contre le Tyran et de l'utopie communautaire) était vouée, dans ce Détour nécessaire à la compréhension des fondations étatiques, à sans cesse se rejouer sous nos yeux, bégayant des structures toujours proches et, pourtant, sans cesse différentes.

Ainsi la rébellion de Taylor établit sa base en pays Dan/Gyo, à cheval sur les frontières $\mathrm{du}$ Liberia et de la Côte d'Ivoire, comme les ethnies en question. De même la base du RUF, à partir de ses appuis libériens, s'installe aux confins de la Sierra Leone. Et, en sens inverse, l'ULIMO, puis le LURD, les mouvements "anti-Taylor ", partent de l'arrière frontière guinéenne, avec l'appui de l'armée de ce pays, et semble-t-il de la CIA, pour occuper l'Ouest du Libéria, et progresser vers Monrovia.

Mais n'est-ce pas là, justement, une vision étatiste, du moins géocentrée, du conflit ? Certes, les frontières artificielles et les ethnies transfrontalières représentent bien les faiblesses de l'Etat, et des opportunités remarquables pour les guérillas. Mais dans cette guerre nomade, justement, se créée une zone transfrontalière spécifique, à cheval sur trois à quatre frontières, hors l'Etat d'abord, puis un espace de «non-Etat ", qui a pourtant ses pouvoirs et ses régulations, comme on l'a vu pour la Sierra Leone.

A l'opposé de ces solidarités intra-ethniques, des rivalités de longue durée opposaient des ethnies voisines : au Libéria, en particulier, les Gyo et les Krahn à la frontière Est, ou les Lorma et les Madigos près de la frontière de la Sierra Leone. Dans ce dernier cas, le sociologue Amos Sawyer ${ }^{25}$ a montré que la rivalité se dit sur le modèle anthropologique oncle/neveu : de même que les relations à plaisanteries entre groupes, ces "patterns " des relations sociales restent très vivaces aujourd'hui. Soulignons aussi qu'une relation moins connue oppose « en tuilage » les côtiers à ceux de l'hinterland, sous l'angle de « ceux qui ont connu le Blanc le premier » (sic!), comprendre «plus civilisés» (re-sic) que ceux de l'intérieur. Aliénation certainement intériorisée, mais qui structure encore des hiérarchies informelles, nourrit des rancœurs, influence ou décourage les unions matrimoniales.

Cependant alliances et rivalités peuvent être très labiles, suivant le modèle fondamental de la segmentarité ; lorsque le conflit tourne avec des groupes différents, dans des agencements changeants, ou passe à un niveau spatial différent. C'est là l'erreur fondamentale d'analystes de la Côte d'Ivoire qui ont voulu établir en modèle idéal-typique rigide l'opposition bété/baoulé qui clivait le pays selon une division Ouest/Est, recoupait leaders et partis (depuis l'époque d'Houphouët à aujourd'hui : PDCI/FPI), mais surtout deux conceptions du pouvoir: étatiste et conservateur vs communautaire et progressiste. Or, l'irruption du «tiers soumis »- les nordistes et le RDR - a démontré que ces antagonismes de premier niveau pouvaient se transformer très rapidement en alliance face à un adversaire commun. Et à vrai dire le jeu des trois blocs ethnico-régionaux ivoiriens, depuis les dix dernières années, semble avoir fait le tour de tous les agencements possibles.

59 La déterritorialisation, elle aussi, a-t-elle une généalogie? Biographiquement, elle semble au contraire sa négation, branchée sur l'évènement premier qui arrache l'individu à l'espace-temps de sa société, et le projette dans la guerre, où sa survivance incertaine le ballote de flux en reflux, de camps en factions opposées, de violences en fuites. "Quand la guerre est arrivée chez nous...»: ainsi commencent d'ordinaire les 
extraordinaires récits de vie recueillis en Sierra Leone et au Libéria. La guerre, présentée comme fortuite et aléatoire, personnifiée mais aveugle, frappe au hasard et brouille d'emblée toute frontière. Les victimes se voient arrachées à leur environnement villageois, projetées dans cette vie inversée qu'est celle de la brousse ${ }^{26}$, à la fois victimes et bientôt acteurs de guerre, passant parfois d'une rébellion à une faction gouvernementale, d'un camp à l'autre. Les frontières d'âge, d'activité quotidienne, de morale ordinaire s'inversent ou se brouillent: si dans son discours adapté au monde de l'humanitaire ou aux normes supposées de la société englobante -, le déterritorialisé se présente toujours comme victime passive, jamais comme acteur de guerre, il est en fait souvent les deux, victime et bourreau, en des proportions de lieux et de temps variables.

60 Ainsi en Sierra Leone, une société se reconstitue, même nomade: guerriers et compagnes, médecins et auxiliaires, enseignants et porteurs, esclaves ${ }^{27}$ des mines de diamants et maîtres de guerre, tueurs et apprentis... Mais si elle est, à bien des égards, une contre-société, elle s'inspire forcément des modèles anciens, jusque dans l'esclavage ou la prédation des sédentaires, faisant revivre d'anciennes mythologies et images du pouvoir: tel Foday Sankoh, représenté en fauve tout puissant sur les peintures naïves des postes du RUF dans la brousse de Kailahun.

61 Arrachement du territoire et brouillage des repères vont le plus souvent de pair, dans la création expérimentale de sociétés perverses dont les enfants-guerriers pourraient être le symbole premier. Dans la référence centrale pour comprendre le rôle des catégories d'âge dans les sociétés régies à l'ordinaire par le « Poro » et plongées dans la guerre, Paul Richards met l'accent sur l'exclusion structurale des plus jeunes, ou du moins leur minorisation sociale par le pouvoir des Anciens; et a contrario sur cette véritable inversion sociale, en un sens carnavalesque, que représente la domination des jeunes « fighters » sur les vieux, dans la parenthèse du conflit. Pour Richards, ces exclus du pouvoir et de la terre forment un groupe turbulent et instable, en rivalité avec leurs aînés pour les biens, les femmes et les postes. Une exclusion que certains sociologues comme $\mathrm{E}$. Terray n'ont pas hésité à formuler en termes de classe dominée ${ }^{28}$.

Mais dans la guerre nomade, des pseudo-familles se constituent où le critère d'âge n'est pas toujours dominant et peut même s'inverser. De même pour les femmes et les filles guerrières, vivant souvent à part dans des contre-sociétés d'amazones. Les catégories sociales de l'initiation, le rapport au sexe et à la violence sont parfois monstrueusement transformés, faisant par exemple de l'inhumanité (par rapport aux valeurs de son groupe d'origine) de l'enfant soldat un guerrier par excellence, dont les mythes d'origine du Tyran rappellent les actes de cruauté.

\section{Autonomisation et médiations de la violence}

Dans les «chroniques de la violence ordinaire » développées dans les années 1990 pour l'Institut français de Polémologie (puis ultérieurement pour le « Centre d'Etudes sur les Conflits »), nous proposions, en nous fondant sur le cas de la Côte d'Ivoire et de l'entropie ouest-africaine, le concept-clef de "continuum de la violence », dont la valeur analytique et même prédictive devait se révéler pour le conflit en cours.

Continuum d'une violence croissante, souvent ignorée, déniée ou forcluse aux observateurs extérieurs, dont nous proposions de distinguer la violence sociétale (dans ses modèles ethnicistes bien particuliers, y compris les «traces de la guerre »), la 
spécificité des violences urbaines, et celle de l'Etat, jusque dans sa dimension symbolique et historique où elle s'origine, et qui la refonde à chaque crise. Analyse que plus tard on a pu appliquer avec profit à l'anthropologie du conflit libérien ${ }^{29}$ (notamment en référant les formes de violence, qui paraissaient inédites, aberrantes, sauvages à bien des observateurs pressés ou moralisateurs - en particulier dans leurs échos journalistiques), à l'historique des guerres ethniques, mais aussi aux rituels initiatiques ou à la criminalité urbaine.

La violence urbaine conserve des conflits ruraux les querelles de femmes, les cambriolages, agressions et vols à la tire comme occasions de lynchages par des voies en général extrêmement brutales : pilonnage par les femmes ou lapidation des voleurs, purges à l'acide, usage de bâtons et de machettes... correspondent à une violence lignagère toujours présente, comme le " pimentage » ou la chicote des récalcitrants, y compris dans le système scolaire. Les "corps habillés" ne sont pas en reste: les bastonnades ou les passages à tabac des commissariats n'étant que l'antichambre de la sinistre MACA (Maison d'arrêt) d'Abidjan.

De « petit métier » le brigand de quartier, dans un rapport de protection/exaction prèmafieux vis-à-vis de ses proches voisins, la délinquance s'est autonomisée dans des lieux et des temps propres: failles urbaines, ponts, marchés, gares, "ghettos " et bidonvilles qui dès la tombée de la nuit sont le cadre d'activité des bandes ou des gangs. L'ivoirisation progressive de la délinquance dans la dernière décennie contredit les stéréotypes l'attribuant de manière privilégiée aux étrangers sahéliens. Une diffusion dans le corps social des contre modèles de délinquance passe par d'autres idéaux du corps (folklore carnavalesque: kaolin, nudité, grimage, alcools et drogues) de la musique, du langage, adoptés par la jeunesse urbaine, notamment estudiantine.

Ces «modèles d'inconduite » se mêlent de manière plus obscure à la toute-puissance du simulacre ("guerriers urbains ») inspirés et de la tradition et des médias, mais aussi des conflits en double d'une sorcellerie toute puissante pour expliquer succès et échecs, $y$ compris des entreprises criminelles. On pourrait d'ailleurs analyser les violences urbaines abidjanaises, par exemple, comme l'Imaginaire d'une guerre toute proche, celle longtemps du Libéria, puis celle de la guerre civile ivoirienne elle-même - le passage au Réel vers la « vraie guerre » étant, dès les débuts, incessant, l'aventure de Charles Taylor (condamné, emprisonné et... évadé aux USA semblant assez archétypale) attirant dès ses débuts toutes les têtes brûlées de la sous-région.

Il était déjà évident, tant dans les réactions populaires que dans de rares et violentes campagnes xénophobes et anti-occidentales du pouvoir d'Abidjan - y compris houphouétiste, ce qu'oublient volontiers aujourd'hui les chantres du "présidentprophète » - que les Européens, et les Français en particulier se trouvaient en fin de liste, boucs émissaires commodes non seulement en fonction de l'histoire mais aussi de leur nombre (à l'époque environ 10000 Français "de métropole» et autant de binationaux) et de leur place provocante dans l'économie urbaine ( $50 \%$ des emplois urbains dépendant de PME françaises), de leur visibilité parfois excessive et en fin de compte de leur vulnérabilité structurelle dans une métropole de 2,5 millions d'habitants (notamment dans l'impossibilité matérielle d'une évacuation massive et rapide, dans le cas d'une population locale hostile, si l'administration ou l'armée ivoirienne étaient complices d'exactions).

69 Mais plus largement, peut-être faudrait-il ici, marquer les bornes du nomadisme guerrier et de la déliaison sociale, et s'inspirant du fameux Discours de La Boétie ${ }^{30}$, 
inverser la question de la violence : d'où vient, dans cette aire ouest-africaine, non que tous soient poussés vers le conflit, mais que des instances sociales, des relations, des lieux particuliers permettent à des individus et des groupes de résister à l'enchaînement entropique, autant de micro résistances au devenir-guerrier qui les menace de toutes parts? Que la guerre, loin de devenir totale ou génocidaire, soit somme toute limitée?

70 Bien des commentateurs critiques des thèses de la segmentarité s'en tiennent, en fait, à l'opposition mécanique et croissante des segments sociaux, négligeant les instances emboîtées de règlements de conflits et d'autolimitation de la violence. Si l'on revient brièvement aux textes fondateurs du modèle de la segmentarité, Evans Pritchard ${ }^{31}$ luimême affirme, dans une curieuse métaphore qu'entre les sections Nuer en conflit «les fibres de parenté et d'alliance, d'affiliation aux classes d'âge, d'intérêts militaires, voire économiques (...) ramènent toujours les communautés l'une vers l'autre ».Comme chez les Guayakis vus par Clastres ${ }^{32}$, le "chef à peau de léopard » des Nuer n'a d'autre pouvoir que celui de médiation en cas de consensus. Tirant son caractère sacré de son association avec les dieux de la terre, ayant le pouvoir de bénir et de maudire, il est respecté par les deux parties en conflit; mais d'autre figures de la médiation peuvent s'imposer, en mineur les devins-guérisseurs, exceptionnellement les Prophètes, au carrefour du religieux et du politique. Et si la Côte d'Ivoire n'est pas (encore ?) le Rwanda ${ }^{33}$, ni dans son ensemble, le Libéria, sauf aux frontières ouest, c'est bien qu'ici quelque chose a tenu du lien social antérieur qui, malgré tout, fait encore sens entre autochtones et allogènes, plutôt d'ailleurs à l'échelon local ou dans les réseaux interpersonnels que dans le champ politique à l'occidentale ${ }^{34}$.

71 Paradoxalement, la zone nord est fort mal connue, malgré la politique d'accueil tout azimut des rebelles envers les médias sympathisants (contrairement aux blocages, voire aux défaillances de la politique progouvernementale): quid de l'épuration ethnique à Bouaké et dans les villages baoulé, des massacres des « corps habillés », des fonctionnaires sudistes et parfois de leurs familles? Cette ville de Bouaké, qui apparaîtra peut-être un jour comme un petit Timisoara ouest-africain de la désinformation: ONG, journalistes et observateurs occidentaux présents, mais inconscients du réel des violences locales... A l'inverse les 100 à 300 manifestants (selon les différentes sources) massacrés le 25 mars 2004, lors d'une manifestation interdite de l'opposition à Abidjan par les "forces de l'ordre ", l'armée et des milices extrémistes semblent bien marquer le passage d'un dernier seuil avant l'extension généralisée de la violence urbaine et entacher gravement la légitimité du pouvoir. Mais pour tenir la balance égale, faudrait-il encore connaitre les milliers de civils massacrés par les rebelles dans la zone frontalière de l'Ouest, dans ce que l'on a pu nommer dès 2002 une libérianisation de la guerre, la situation d'Abidjan rappellerait plus, s'il fallait un rapprochement africain, le « modèle congolais ». Enfin ne faudrait-il pas parler, au-delà de son asymétrie Nord/Sud, d'une surmédiatisation de la guerre ivoirienne, davantage due à son importance stratégique pour l'influence française et ses quelques 4500 hommes engagés, si on rapporte ce conflit somme toute encore très limité aux quelques trois millions de morts des guerres congolaises.

D'autant que les nordistes sahéliens - migrants burkinabés et maliens principalement représentent à peu près une population de cet ordre, et que s'ils ont été marginalement inquiétés, parfois tués ou chassés au plus fort de la guerre, leur présence à terme semble pourtant assurée, la terre pour les vivriers systématiquement accordée malgré 
des « néo-règlements » discriminatoires ${ }^{35}$. Si les relations entre groupes ont tenu, c'est que loin d'être une instance fictive ou intégrée, la communauté villageoise comporte certains rituels, médiateurs, mécanismes de régulation et d'accueil, ramenant une paix relative après chaque crise.

De ces prémisses d'une anthropologie de la paix en société segmentaire, peut-on établir des parallèles avec les sociétés ouest africaines? Si ces peuples pris dans la guerre nomade ont tous des traces et des rituels hérités des guerres anciennes, ils réactivent, ou réinventent des médiations plurielles, aujourd'hui partout à l'œuvre en Côte d'Ivoire, mais aussi en situation dite de "post-conflit » comme en Sierra Léone ${ }^{36}$. En ce qui concerne les processus ethniques de paix et de réconciliation ivoiriens, l'alliancereprésente dans toute la zone culturelle le modèle rituel dominant, comme paix et union des communautés en conflit.

74 Une typologie rapide des médiations «néocoutumières " peut s'inspirer de ce qui s'est passé, sur le plan militaro-politique dans les crises des dernières années, et des médiations plus ordinaires entre communautés et Etat (conflits fonciers, développement, etc.). Il faut distinguer les cérémonies nationales, à la fois ritualisées et personnalisées, des médiations ordinaires où les représentants locaux de l'Etat inventent des formes syncrétiques du politique. Un des rituels ivoiriens, utilisé par tous les présidents successifs, est de passer par les représentants de chaque ethnie (pudiquement nommée selon la région) pour organiser de grandes messes radiotélévisées, entre allégeance et propagande. Quand une crise grave survient, ces élites, les élus, les " chefs spirituels », les autorités locales (chefferies, "rois »...) sont aussi mis discrètement à contribution pour des médiations en direction des communautés ou des familles. Par exemple, cela a été les cas après les différents "complots» de la période houphouétiste, mais a échoué en direction des bété du Guébié, massacrés par milliers lors de la guérilla de Kragbé Gnagbé, en 1970 ${ }^{37}$.

Par opposition à ces discrètes médiations, qui se passent en secret lors de toute crise politique, les « tournées en brousse » de l'époque coloniale se sont transformées depuis Houphouët en grandes cérémonies néo-coutumières, où les représentants locaux coutumiers sont associés aux cadres urbains de la province, pour des prises de décision majeures ; par exemple pour lancer de grands projets de développement ou des réorganisations administratives: à cette occasion, le chef d'Etat, les ministres, se déplacent, avec le maximum d'écho médiatique, à l'image d'une "Société de cour " itinérante.

76 Le « modèle » d'une médiation réussie semble être ce fameux "dialogue à l'ivoirienne ", qui s'ancre sur des conceptions ethniques de la négociation. Redondant sous Houphouët, où il participait d'une " culture de la paix » avec son président-prophète, ce dialogue est bel et bien passé dans les relations sociales et interindividuelles: il constitue certainement un des mots-clef de toute négociation et explique en partie pourquoi les évènements récents n'ont pas dégénéré en guerre civile totale. Rappelons que le déroulement de ce "dialogue " suppose qu'un arbitre jugé impartial demande successivement la version de chacune des deux parties, avant de lancer la discussion, puis rapproche les points de vue et propose une solution.

77 Très souvent, le préfet, et plus encore le sous-préfet (populairement «le commandant»), a repris, de l'attirail des fonctions du commandant de cercle colonial, le rôle de médiation dans les affaires villageoises. Et ce, dans les domaines les plus inattendus, par rapport à leur fonction officielle: querelles de femmes ou de voisinage, de 
tractations foncières en particulier, où ces autorités locales délivrent en dehors de toute légalité nationale des " attestations » au statut ambigu - lors d'achat/vente entre autochtones et immigrés par exemple - qui, répondant à la demande sociale d'une médiation extérieure aux deux parties, a pourtant « force de loi », du moins localement. En d'autres termes, ce rôle de médiation est inscrit en creux dans les attentes sociales. Or, si cette fonction est possible en forêt, entre autochtones et allogènes notamment, il ne saurait en être question dans le Nord, où tout sudiste et représentant de l'administration a été chassé ou supprimé.

D'un côté, la communauté villageoise se rétracte, fuit, devant toute intervention extérieure, en particulier devant l'épreuve de force en face des appareils d'Etat; d'un autre côté, en y mettant les formes coutumières, le médiateur d'Etat peut participer d'une certaine pluralité des pouvoirs (théorie de l'« empilement $»^{38}$ ), assez syncrétique dans ses manifestations.

On pourrait insister sur une catégorie très intéressante de notables, internes/externes, que sont les ressortissants, cadres et élus locaux. Comme on le sait, les villages sont organisés, à Abidjan, en " associations de ressortissants ${ }^{39}$ qui cotisent et agissent pour et dans le village : même si l'observateur extérieur n'en a pas conscience lors de sa visite, ce «double villageois » est extrêmement actif et puissant: si les cadres ne sont pas là lors de la négociation, l'affaire est mal partie et l'accord risque d'être vite remis en question.

On a cité la fonction informelle de "chef spirituel », qui comme à l'époque d'Houphouët, sert de répondant pour un groupe ou une ethnie, à Abidjan. Plus formel, l'appui de chef religieux peut être recherché, bien que le clivage Nord/Sud ne doive surtout pas être placé sur ce plan, et qu'inversement un évêque ou un imam ne puisse agir hors de son aire d'influence. Cependant, l'intervention conjointe de différents dignitaires religieux peut se révéler efficace, en raison même de l'influence des mouvements prophétiques et syncrétiques en Côte d'Ivoire.

81 Ceci dit, l'instrumentalisation des rituels et procédés autochtones par la société englobante, fut-elle armée des meilleures intentions du monde, est souvent périlleuse et parfois contre-productive: rien de bien différent des mêmes tendances qui s'observent à travers l'idéologie et la praxis du «développement» ou de «l'humanitaire », ces « croyances occidentales » selon Rist ${ }^{40}$, toujours en porte-à-faux devant le retrait communautaire. Et il faut, par ailleurs, se garder d'idéaliser la riche sociabilité des associations ouest africaines: si l'on a pu montrer la vitalité des associations urbaines abidjanaises, avec la floraison par exemple des "rosicruciens", des loges maçonniques et bien sûr des divers prophétismes, syncrétismes ou mouvements religieux d'origine protestante comme substitut urbain à la perte - voire à la dette - communautaire, la situation libérienne en montre un versant beaucoup plus sombre.

En effet, si les diverses associations (ressortissants, cultuelles, épargne, etc.) jouent, comme on le sait, un rôle d'intégration et cherchent sans cesse à reconstituer en ville la " communauté perdue » sur d'autres bases, certaines réinterprétations peuvent donner des résultats bien plus déstabilisateurs, vers l'extérieur; c'est ainsi qu'au Libéria, où pendant la guerre Monrovia constituait le "paradis des sectes», les «sociétés secrètes", anciennes et contemporaines ont longtemps été interdites, car véhiculant des contre-valeurs nativistes, contre le pouvoir congo et l'occidentalisation officielle. C'était bien le cas de toute manifestation de la "Poro Society", jusqu'à ce que le 
pouvoir les folklorise et les intègre, le président Tubman lui-même clôturant en 1978 les festivités comme grand dignitaire - ce qui n'empêche pas l'actuel revivalisme d'un Poro de brousse cherchant à contrôler à nouveau ces jeunes combattants «ensauvagés, car n'étant pas passés sous le joug de la Loi », selon la forte parole de nos interlocuteurs de l'Eastern Province de Sierra Leone.

83 Mais des sociétés secrètes urbaines (même si certaines avaient des racines ethniques fortes) ont connu, dans le cas du Libéria, des dérives quasi-criminelles, telles celles dénommées « Léopard », « Nigi », « Susha », « Joyn », « Kela », etc. qui s'adonnaient à de sanglants assassinats externes et à des règlements de compte internes, sur le modèle sorcier où chacun doit à son tour fournir une victime) et selon des formes atroces et spectaculaires de meurtres propres à terrifier et à frapper les imaginations. Un observateur du politique dans le Comté de Grand Bassa ${ }^{41}$ notait que la technique de la violence reste la même entre règlement de compte des sociétés secrètes et liquidations mafieuses ou politiques: des "façons de tuer soudaines, brutales, imprévisibles». Cette spectacularisation interne, autochtone, de la terreur politique s'articule d'ailleurs aux techniques de guerres (chimères, dépeçages des corps de l'ennemi), créations de situations et de vidéos "gore» comme le fameux documentaire du chef de guerre Prince Johnson filmant l'exécution « live » de l'ex-chef d'Etat Samuel Doe.

Plus certainement, la projection (en tout sens) de films de guerre occidentaux et orientaux avant les combats, mêlés aux techniques de contrôle par l'alcool et les drogues, a participé à une certaine déréalisation ou virtualité d'une guerre nomade très syncrétique, parcourant aussi de nouveaux territoires entre Imaginaire et Réel prolongement, au fond, d'une guerre en double qui passe volontiers par la sorcellerie (ju-ju et talismans, sacrifices et instrumentalisation des organes des cadavres, croyance à l'invincibilité et à l'envoûtement de l'adversaire) dont même la Force Licorne a pu vérifier qu'elle était militairement efficace car socialement partagée. Si «criminalisation du pouvoir » il y a, c'est dans le sens d'une gouvernementalité multiple, où bas et haut partagent bien les mêmes modèles de violence et de conquête du pouvoir, en tout cas pas un phénomène déviant résultant d'une évolution récente et mafieuse, si ce n'est par interprétation transfrontalière et branchement sur des diaspora ou réseaux transnationaux.

Nous avions déjà remarqué les liens entre cette sociabilité en quelque sorte sectaire et des traits accentués d'un néo-patrimonialisme porté dans l'arène politique. Les partis libériens en portent la marque : base ethnique et sociale restreinte, initiation par la violence, ambitions et "politique du ventre " exacerbées, idéologie et programmes inexistants... La guerre nomade opère la continuation élargie d'un socius conflictuel par d'autres moyens, offre à cette violence et à ces conceptions brutales du politique un plus large champ, en conserve les formes, renvoyant le trauma fondateur des capitales coloniales et de leurs élites monopolisatrices de pouvoirs et de richesses vers une lutte démultipliée entre acteurs et communautés.

\section{Régimes du politique et sociétés de guerre}

Si l'Etat est donc bien une idée neuve, dans bien des temps et des lieux, n'est-il pas aussi refusé et déjà obsolète dans cette Afrique de l'Ouest où s'autonomise la violence d'une guerre nomade? Pour autant, ne peut-on observer des déclinaisons en système, des 
modalités de la violence, et des rapports complexes entre ethnicité et appareils d'Etat que nous proposons de conceptualiser comme « régimes du politique »?

Pour ne prendre en exemple que les quatre «Etats-Nation » concernés par la guerre nomade ouest-africaine, ces régimes du politique, alliés à une histoire spécifique aboutissent à des dispositifs ethnico-politiques intéressants. On commence à peine à s'intéresser à leur interrelation, à leur hiérarchisation, et à ce que le géographe Jean Gallais nommait, dans un article novateur ${ }^{42}$, des «pôles ethnopolitiques».

Ainsi la Côte d'Ivoire apparaît comme un curieux laboratoire des thèses de Gallais dans la mesure où, au-delà des " 60 ethnies » de la doxa administrative, sa tripartition actuelle correspond bien à de grands pôles ethno-politiques. Ainsi, autour de la prépondérance baoulé, s'organise le groupe akan, tandis que le monde krou s'est longtemps reconnu dans les aspirations bété, et qu'enfin le monde sénoufo-malinké s'est imposé en tiers (l'opposition latente entre les deux groupes nordistes apparaissant actuellement dans les règlements de compte entre factions rebelles); mais l'expérience ivoirienne a ceci de fascinant qu'il y a eu simultanément projection dans le registre du «politique à l'occidentale» - partitaire, électif -, dans la formation des trois grands partis, PDCI , FPI et RDR, incarnés par les trois hommes politiques majeurs du drame politico-militaire.

Non seulement cette tripartition des régimes du politique n'est justifiée que de manière probabiliste, mais elle offre une base géopolitique à des réorganisations permanentes, enfin la situation urbaine et le conflit lui-même offrent des occasions multiples d'échapper à un schéma cependant très éclairant.

Avec ses quatre grands groupes ethnico-régionaux, on pourrait s'attendre à ce que la Guinée retrouve des agencements labiles, comme en Côte d'Ivoire ; il n'en est rien, car au-delà du faible poids des Forestiers (agrégat quelque peu artificiel lié au milieu naturel), le dualisme exacerbé entre pouvoir soussou de Conakry et challengers malinkés (représentés par la forte personnalité du chef de l'opposition Alpha Condé) est en fait médiée par le fort groupe peulh, identifié à une forte et originale culture, à un secteur économique, et au fief du Fouta Djallon. Ceci dit, la logique actuelle de l'alternance propose effectivement un chef de l'opposition qui s'extrait du carcan ethnique pour revendiquer un électorat transrégional, tandis que chaque parti est passé maître dans l'instrumentalisation des adhérents d'ethnies minoritaires ${ }^{43}$.

Dans la Sierra Leone proche, en revanche, les jeux de l'ethnicité sont à la fois plus complexes et bien moins déterminants dans l'histoire des régimes du politique, bien que pour des observateurs avertis, le pouvoir actuel soit en apparence surtout mendé (sur le fond, aux mains des élites crios), ce qui n'empêche pas des sortes d'adoption croisées de leaders politiques par des blocs extérieurs à leur propre origine. Par contre, tout à la fois en fonction des diamants et des frontières, l'opposition des peuples Kono du district de Koïdu et des Mendéet Kissi de celui de Kailahun fait sens en ce que ces derniers forment la base spatiale et ethnique originelle du RUF de Foday Sankoh, tandis que les premiers représentent le fief où sont nés les Kamajors - et, bien sûr, les deux s'opposant historiquement à la domination des Crios de Freetown ${ }^{44}$. Quant au Libéria proche, dont on a vu la double articulation du politique, à partir de deux micro-ethnies frontalières (Krahn et Gyo/Mano) et de ligues duelles (voir supra), on peut remarquer les oppositions croissantes autour, par exemple, du cas madigo (aujourd'hui représenté par le LURD) qui ne peut espérer, malgré l'appui américain et libérien, revendiquer longtemps le pouvoir à Monrovia. 
92 On pourrait analyser ainsi, de proche en proche, les régimes du politique ouestafricains et leurs agencements contingents, depuis le noyau ethnicorégional mossi, au Burkina Faso, jusqu'au dualisme Nord-Sud d'Etats côtiers comme le Bénin et surtout le Togo et discerner dans ces fractures sous-jacentes les futures lignes des conflits à venir. A cette idéelle géopolitique interne, on opposera les fluctuations accélérées de l'ethnicité et des alliances, ce que le cas ivoirien a bien montré au détriment de la rigidité des analyses en termes d'opposition Ouest-Est, qui constituent sur ce clivage temporaire des idéaux-types du politique devenus obsolètes. Mais l'exemple sénégalais serait pertinent à plus d'un titre: d'une part, à cause de la «wolofisation $»^{45} \mathrm{du}$ politique et du social autour du groupe dominant ; et, surtout, parce que leproblèmedes castes y sembleplus marqué que celui de l'ethnicité,obligeant à reconceptualiser autour d'autres facteurs les régimes du politique, ne serait-ce qu'à la marge.

93 Ainsi, plus que des cartographies ethniques figées dans les siècles passés, cette articulation dynamique, au sens de Balandier, fait apparaitre môles de résistance, clivages et fractures, mécanismes de transmission transfrontaliers et entropie croissante autour de foyers initiaux restreints. La déconstruction de ces dynamiques lors des périodes de pacification relative.

Plus qu'une microsociologie des groupes combattants, ou une analyse criminalisante, c'est donc de ces déséquilibres profonds qu'il convient de tenir compte pour comprendre la guerre nomade et la régulation de ces conflits nomades pourrait s'en inspirer. Toutefois, dans ces processus machiniques où les acteurs sont inconsciemment déterminés, il n'est pas sûr que le "sur-Etat mondial» y puisse quelque chose. En particulier en termes de rééquilibrage des territoires et des générations, sinon comme répressions et contraintes - tout comme le Poro et ses échecs à l'échelon communautaire? Et surtout si les «machines désirantes » de la guerre n'ont d'autre projet que de se perpétuer, en termes de machine de guerre nomade, et en tant que désir de mort.

Les dieux du bush ont-il encore soif ? Peut-être faudrait-il voir, comme une parabole des guerres à venir, le culte d'un héros guerrier divinisé, en pays gyo, dans le Comté de Nimba, sur cette frontière poreuse entre Libéria et Côte d'Ivoire d'où est parti le conflit il y a quinze ans. Devenu le masque "T'To Ge", d'après le nom du héros défunt ("T’to »), le Dieu de la guerre demande sans cesse des sacrifices, à la veille du combat, et le Maître de guerre est respecté à la mesure des meurtres qu'il a commis ${ }^{46}$. L'irruption, dans ce Comté de Nimba hanté de l'esprit des guerres anciennes, d'une violence d'Etat incontrôlée correspondait bien à l'image qu'avaient les autochtones d'un pouvoir tyrannique et de sa violence sauvage, mais aussi de la guerre totale à mener. Depuis, la violence a passé les frontières, s'est déchaînée et continue à nomadiser : le dieu «T'to Ge » - ou ses avatars - revit, gagne des fidèles, cherche des victimes, conquiert les esprits, gagne de nouveaux territoires... 


\section{NOTES}

1. Richards P., Fighting for the rain forest, Oxford, James Currey, 1996.

2. Revolutionary United Front.

3. Bateson G., La cérémonie du Naven, Paris, Editions de Minuit, 1971.

4. Clastres P., Recherches d'anthropologie politique, Paris, Seuil, 1980 ; Deleuze G., Guattari F., Mille Plateaux, Paris, Editions de Minuit, 1980.

5. Taîbo P.I., L'année où nous étions nulle part, Paris, Métailié, 1995.

6. Vidal Naquet P, Le Chasseur Noir, Paris, La Découverte, 1992.

7. National Patriotic Front Of Liberia.

8. Galy M., « Les avatars de la Dcgtx: agence technique ou gouvernement-bis? », Politique Africaine, Décembre 1993, N52, pp 135 -139.

9. Liberians United for Reconciliation and Democracy.

10. Legendre P., Jouir du pouvoir, Paris, Editions de Minuit, 1976.

11. Concept que retrouve Anna Tsing pour les marges d'Asie du Sud-Est: In the Realm of the Diamond queen, Princeton, 1983, cité par J. F. Bayart dans un article combatif intitulé «Le crime transnational et la formation de l'Etat », Politique africaine, n93, mars 2004, pp.93-105.

12. Gberie L., War and peace in Sierra Leone, Montréal, Partnership Africa Canada, 2002.

13. Igué J., L'Etat - entrepôt, Paris, Karthala, 1992.

14. Mouvement patriotique de Côte d'Ivoire ; Movement for Democracy In Liberia: comme pour nombre de partis, la dimension dérisoire des «mouvements rebelles » rejoint celle des noms de guerre...

15. Gellner E., Arabs and berbers, from Tribe to Nation, Londres, Duckworth, 1972.

16. Marie A., "Une anthropologique communautaire à l'épreuve de la mondialisation », Cahiers d'Etudes Africaines, 166, 2002.

17. Voir en particulier sa remarquable analyse $d u$ «Plan foncier rural » et de la situation foncière actuelle à la lumière, bien sombre, de l'ivoirité: « La question foncière en Côte d'Ivoire ", Politique africaine, $\mathrm{N}^{\circ} 78$, Juin 2000, pp. 94-125.

18. Voir l'ensemble du numéro de Critique internationale: "problématiques de l'autochtonie ", $n^{\circ} 10$, janvier 2001.

19. Etienne P., "Le fait villageois baoulé », in "Communautés rurales et paysanneries tropicales", Orstom, Paris, 1976.

20. Chauveau J.P., ibid.

21. Voir les thèses d'A. Appaduraï, Après le colonialisme, Paris, Payot, 2001, qui s'appliquent particulièrement aux déterritorialisations villageoises.

22. Parti Démocratique de Côte d'Ivoire, section du Rassemblement Démocratique Africain : le plus ancien des partis ivoiriens (dont le leader actuel est l'ex-président Henri Konan Bédié) forme avec le Front Populaire Ivoirien de Laurent Gbagbo et le Rassemblement des Républicain d'Alhassane Ouattara les pôles du «triangle ethno-politique » ivoirien (avant le bouleversement des mouvements rebelles et patriotiques) dont nous avons dit possibles toutes les combinatoires duelles, d'alliance ou de rivalités.

23. Boétie E. de la, Le Discours de la servitude volontaire, Paris, Payot, 1976 ; G. Deleuze, 1000 Plateaux, op. cit.

24. Kaplan R., « The coming anarchy », Atlantic Monthly, février 1994, vol.273, n², pp. 44-76.

25. Sawyer A., "Sharing the kola nut », Monrovia, Center for Democratic Empowerment, 2000.

26. Voir à ce sujet Cartry M, La fonction symbolique, Paris, Gallimard, 1979. 
27. Voir les thèses de Rosalind Shaw, Memories of the Slave Trade, Chicago, University of Chicago Press, 2002 sur le revival de l'esclavage durant les guerres sierra léonnaises, quelques soient les factions combattantes.

28. Richards P., op.cit. ; Terray E., Le marxisme devant les sociétés primitives, Paris, Maspéro, 1969.

29. Galy M., «Les mouvements religieux et les formes de la violence à Abidjan », Paris, Institut français de Polémologie, 1988 ; et Galy M., «Libéria, machine perverse », Cahiers d'études africaines, $\mathrm{n}^{\circ} 150-152,1998$, pp. $533-553$.

30. Boétie E. de la, Le Discours de la servitude volontaire, op. cit.

31. Evans-Pritchard E., Les Nuer. Description des modes de vie et des institutions politiques d'un peuple nilote (1937), traduction française, Paris, Gallimard, (1969), collection Tel, 1994.

32. Clastres P., Chroniques des Indiens guayakis, Paris, Plon, 1972.

33. Au-delà de l'extraversion particulièrement marquée du champ politique ivoirien, notamment dans l'influence des représentations extérieures de la violence politique, on ne peut s'empêcher ici de relever l'ambiguïté de cette référence rwandaise heureusement encore lointaine : s'il est certain que des intervenants extérieurs (France, Union Africaine) se sont interposés avec cette référence en tête - et celle de leurs propres insuffisances en 1994, la doxa journalistique occidentale - et ses représentations outrées, pour ne pas dire, pour certains « observateurs " pas forcément désintéressés, systématiquement pro-rebelles, la charge systématiques de chercheursacteurs - comme au Rwanda ou au Burundi ?, l'implication spectaculaire d'ONG partisanes - voir à ce sujet les outrances de "Stop-génocide ", ont pu conduire à des prophéties auto-réalisatrices et accélérer les fractures communautaires.

34. Sur cette dualité structurelle d'un champ politique "à double référent ", voir Galy M., «Le Savoir de l'Occident? Sur les représentations du «Coup d'Abidjan », Cultures et conflits, $\mathrm{n}^{\circ} 37$, Automne 2001, pp.105-122.

35. Il ne s'agit pas de nier les embrasements réguliers, mais on le répète, encore limités, des bandes de jeunes autochtones d'autant plus revendicatifs que la guerre civile les a ramenés au village : on est pourtant loin d'une gestion de l'immigration par pogromes et déportation aux frontières selon le contre-modèle nigérian par exemple. Un des paradoxes est que l'exode massif des sahéliens déstabiliserait à coup sûr leur pays d'origine, notamment le Burkina, dont le régime a joué depuis 1989 le rôle de boutefeu en Afrique de l'Ouest, en armant les rébellions successives, y compris ivoiriennes - en échange du pillage des matières premières et de trafics en tous genres. 36. Sur ce point, on verra avec intérêt le cas de l'instrumentalisation des « Paramount Chiefs » par le pouvoir britannique des « Civils Affairs ».

37. Voir un témoignage engagé récent : Gadji Dagbo J., L'affaire Kragbé Gnagbé, Abidjan, NEI, 2002.

38. Thèse notamment de Biershenk T., Les pouvoirs au village, Karthala, 1998. Pour les villages béninois, qui ne semblent pas prendre en compte l'antagonisme des deux formes politiques, communautaire et étatique - quelles que soient les transactions à la marge des réseaux qui les relient.

39. Associations dont déjà Jean-Marie Gibbal avait relevé l'importance et la complexité dès les années 1960 : Citadins et villageois dans la ville africaine, Paris, Maspéro, 1972.

40. Rist G., Le développement, histoire d'une croyance occidentale, Paris, Presses de Sciences-Po, 1996.

41. Genevray J., Eléments pour une Monographie du Comté de Grand Bassa, Dakar, Ifan, 1952.

42. Gallais J., « Pôles d'Etat et frontières ", Cahiers d'Outre Mer, n³5, 1982.

43. Voir l'excellente analyse de Barry A. B., Les violences collectives en Afrique, Paris, L'Harmattan, 2000.

44. Pour la Sierra Leone, voir Ferme M., «La figure du chasseur et les chasseurs-miliciens dans le conflit sierra léonais», Politique africaine, $\mathrm{n}^{\circ} 82,2001$.

45. Selon Diouf M., "Sénégal, les ethnies et la Nation », Dakar, Nouvelles éditions africaines du Sénégal, 1998.

46. Voir Harley G.W. et J. Schwab, Masks as agents of social control, Cambridge, 1950. 


\section{RÉSUMÉS}

Déconstruire les descriptions " réalistes " d'une guerre étatique, pour le cas du conflit ouestafricain qui tourne entre quatre pays depuis 13 ans, c'est s'intéresser à ses espaces transfrontaliers, aux jeux de l'ethnicité et du foncier, à la sociologie mouvante des groupes guerriers. Forger le concept de "guerre nomade ", à l'inverse, c'est faire le choix de suivre des mécanismes relativement autonomes de propagation des conflits, d'insister sur les transformations des " sociétés en guerre ", redécouvrir d'autres instances autochtones du politique comme les communautés, retrouver la dynamique du " continuum de la violence » avant le conflit et les permanentes mutations politiques durant la guerre.C'est donc, dans ce terrible laboratoire politologique, retrouver du sens aux actions des groupes nomades, comme mode de vie, gestion des corps et de la violence ; et dans ces systèmes de guerres interminables, malgré tout, des conceptions autres du politique bonnes à penser, pour la guerre comme pour la paix, mais aussi pour repartir de cet ailleurs si proche pour questionner nos propres devenirs.

In the case of the West African conflict, which four states have been enduring for thirteen years, deconstructing the "realist " descriptions of a State war means taking interest in its transborder spaces, in the ethnical and land ownership interplay, and in the changing sociology of war groups. On the contrary, moulding the concept of a "nomadic war" means following the relatively autonomous mechanisms of conflict spreading, insisting on the transformation of the "societies at war", rediscovering other autochthon political authorities such as communities, and recalling the pre-conflict "violence continuum" dynamic and the permanent political changes that occur during war. In this terrible politological laboratory, the author restores a meaning to the actions of nomadic groups considered as lifestyles, or body and violence management, and, in these never ending war systems, he restores a meaning to other conceptions of the "political».

\section{INDEX}

Mots-clés : Conflit, Afrique de l'Ouest, guerre nomade, violence

\section{AUTEUR}

\section{MICHEL GALY}

Michel Galy est chercheur au CREC (Centre de recherches de St Cyr-Coëtquidan) et au Centre d'études sur les Conflits. Il enseigne les Relations Internationales à l'IEP de Paris. 